Cahiers de recherches médiévales

\title{
Venenum sub melle latet
}

L'image du poison dans le discours anti-hérétique au Moyen Âge

\section{Florence Chave-Mahir}

\section{(2) OpenEdition \\ Journals}

Édition électronique

URL : https://journals.openedition.org/crm/11513

DOI : $10.4000 / \mathrm{crm} .11513$

ISSN : 1955-2424

Éditeur

Honoré Champion

Édition imprimée

Date de publication : 15 juin 2009

Pagination : 161-172

ISSN : $1272-9752$

Référence électronique

Florence Chave-Mahir, "Venenum sub melle latet», Cahiers de recherches médiévales [En ligne], 17| 2009, mis en ligne le 15 juin 2012, consulté le 15 décembre 2022. URL : http:// journals.openedition.org/crm/11513; DOI : https://doi.org/10.4000/crm.11513 


\title{
RM
}

\section{Venenum sub melle latet. L'image du poison dans le discours anti-hérétique au Moyen Âge}

\begin{abstract}
Toxic metaphors are frequent in polemical texts against heresy from the $11^{\text {th }}$ and $12^{\text {th }}$ centuries. These metaphors derive from ancient polemical literature. Metaphors of poison are used to counter heretics, sinners and other deviants and to maintain the unity of the Church in times of ferment.

Résumé: Dans les textes polémiques contre l'hérésie des XI et XII siècles, les métaphores toxiques sont fréquentes. Elles sont issues de la littérature polémique antique. Ces métaphores qui utilisent l'image du poison sont employées pour rejeter les hérétiques, les pécheurs et d'autres déviants et pour maintenir l'unité de l'Église en des temps de contestation religieuse.
\end{abstract}

Dans le sermon qui inaugure sa traque contre les hérétiques d'une vallée piémontaise en 1373, l'inquisiteur dominicain Thomas de Casacho a recours à quelques images saisissantes destinées à dénoncer le venin hérétique, veneno heretice pravitatis. Les herbes épineuses qui prolifèrent, la malice du serpent antique et son venin, les loups infernaux sont autant de métaphores qui désignent ceux, immondes, qu'il convient de combattre ${ }^{1}$. Thomas de Casacho n'innove pas dans ce registre à la fois animalier et diabolique, il ne fait que se conformer à une tradition ancienne qui vise à faire des hérétiques ou des déviants, les habitants d'un inframonde à la lisière de l'humanité.

Tout un répertoire d'images servait en effet aux polémistes du Moyen Âge à évoquer les hérésies. Nous l'envisagerons surtout à travers quelques exemples choisis dans diverses sources qui abordent la question de l'hérésie pour la dénoncer aux $\mathrm{XI}^{\mathrm{e}}$ et XII ${ }^{\mathrm{e}}$ siècles : les Histoires de Raoul Glaber, la correspondance de Pierre Damien, ou dans le Contra Petrobrusianos de Pierre le Vénérable. Là, le discours anti-hérétique se construit autour d'un essaim d'images négatives qui se superposent sans craindre la redondance. Le poison de l'hérésie y occupe une place importante parmi les métaphores relevant du monde animal et des démons ${ }^{2}$. Dans le

\footnotetext{
${ }^{1}$ Nos, frater Thomas de Casacho ordinis Fratrum Predicatorum inquixitor pravitatis in provincia Lombardie superioris et marchia Ianuensi a Sede Apostolica deputatus, cupientes spinas et velpes subortas in agro Dominico quasque malitias serpentis antiqui ignibus instinguibilibus tradere, disponebat, utpote veneno heretice pravitatis infactas igne Spiritus Sancti per compunctionem in rosas et lilia communtari ovesque errantes, quas lupi infernales de ovili dominici gregis subtraxerant a manu pastoris, ad ovile et consorcium et unitatem fidelium Christi redducere et doctrinam sacrilegiam detegendo sanctam fidem catholicam eminencius... dans G. G. Merlo, Eretici e inquisitori nella societa piemontese del trecento, Torino, Claudiana, 1977, p. 259.

2 Voir E. Mitre Fernandez, «Muerte, veneno y enfermedad, metaforas medievales de la herejia », Heresis 25 (1995), p. 63-84, et F. Collard, Le crime de poison au Moyen Âge, Paris, Puf, 2003, p. 178.
}

Cahiers de Recherches Médiévales, 17, 2009 
prolongement de ces discours directement orientés contre les hérétiques, les sermons du XIII ${ }^{\mathrm{e}}$ siècle emploient la même métaphore à propos des pécheurs. Ces images introduisent dans ces sources un écart entre la réalité des apparences et la véritable nature des personnes qu'il s'agit de dénoncer. Ce procédé qui correspond au travail de fiction «comme ouverture d'une représentation sensible dans le discours» comporte une intention précise et, en l'occurrence, « il n'est pas de métaphore innocente $»^{3}$. De nombreux travaux historiques permettent de comprendre ce discours comme une construction inspirée de l'hérésiologie de la fin de l'Antiquité et en partie destinée à fonder l'image négative des hérésies, des déviants ${ }^{4}$ et même des pécheurs. Le venin de l'hérésie ne fait pas seulement référence à la toxicité des doctrines et des personnes, il renvoit aussi explicitement au monde du démon à qui sont automatiquement associés tous les hérétiques. Déviants, pécheurs, voire juifs ou musulmans sont tour à tour diabolisés et indirectement liés au pire des crimes possible quand ils ne sont pas accusés de l'avoir perpétrés. Ces êtres vénéneux ne sont-ils pas comme des serpents qui tentent et sèment le mal ? Ne peuvent-ils pas être comparés au diable qui s'empare des corps, les possède et les dénature ?

Il convient d'examiner les emplois de cette image vénéneuse dans divers discours polémiques pour tenter de la relier à la tradition de l'hérésiologie de la fin de l'Antiquité ainsi que dans quelques sermons plus tardifs.

\section{Les métaphores vénéneuses dans les discours antihérétiques des $X I^{e}-X I I^{e}$ siècles}

Le venin de l'hérésie est un topos de la littérature narrative et polémique des $\mathrm{XI}^{\mathrm{e}}$ et $\mathrm{XII}^{\mathrm{e}}$ siècles, en particulier dans les Histoires de Raoul Glaber, rédigées entre 1030 et 1040. Raoul raconte ainsi comment l'hérétique Leutard fut attaqué par un essaim d'abeilles, animaux vénéneux, et comment cette attaque provoqua en lui un coup de folie qui se traduisit par l'abandon de sa femme et la destruction de l'image du Christ dans l'église. Interrogé par l'évêque Gibuin l'Ancien, Raoul ajoute que Leutard chercha à dissimuler le venin de ses forfaits (venenum sue nequitie). ${ }^{6}$. Ainsi, la piqûre venimeuse des abeilles semble avoir provoqué son hérésie, sa folie, et même la possession diabolique. Ailleurs, Raoul raconte l'histoire de la femme venue d'Italie qui distilla son hérésie en 1022 à Orléans. Elle est qualifiée de possédée du démon (diabolo plena) et l'auteur des Histoires ajoute qu'elle «y demeura quelques

\footnotetext{
${ }^{3}$ P. Ricœur, La métaphore vive, Paris, Seuil, 1975 ; E. Bordas, Les chemins de la métaphore, Paris, Puf, 2003, p. 125.

4 Voir: Inventer l'hérésie? Discours polémiques et pouvoirs avant l'Inquisition, dir. M. Zerner, Nice, 1998 (Collection du Centre d'Études Médiévales de Nice 2) ; D. Iogna-Prat, Ordonner et exclure. Cluny et la société chrétienne face à l'hérésie, au judaïsme et à l'Islam 1000-1150, Paris, Aubier, 1998 et, dans le sillage de ces travaux, U. Brunn, Des contestataires aux «cathares». Discours de réforme et propagande antihérétique dans les pays du Rhin, de la Meuse avant l'Inquisition, Paris, Institut d'Études Augustiniennes, 2006.

${ }^{5}$ Le crime de poison relève de l'indicible, F. Collard, Le crime de poison, op. cit., p. 137 et suiv. ; sur la notion, J. Chiffoleau, «Dire l'indicible. Remarques sur la catégorie du nefandum du XII ${ }^{\mathrm{e}}$ au XV $\mathrm{XV}^{\mathrm{e}}$ siècle », Annales ÉSC (1990), p. 289-324.

${ }^{6}$ Raoul Glaber, Histoires, livre 2, 11, trad. éd. M. Arnoux, Turnhout, Brepols, 1996, p. 135.
} 
temps, infectant de très nombreuses personnes du venin de sa perversité. Et ceux qui avaient reçu cette funeste semence s'efforçaient autant qu'ils le pouvaient de la répandre sur d'autres, plus nombreux encore ${ }^{7}$. Le terme de semence suggère l'ambiguïté d'un poison capable de se transformer en substance qui génère la vie. Un venin transformé en semence n'est pas seulement un paradoxe, il indique la perversité de l'hérésie et sa dimension diabolique ${ }^{8}$. Cette propagation irrémédiable et sans limites rappelle la proximité entre les substances toxiques et les virus qui infectent parfois à mort les organismes. Ainsi, dans les Histoires de Raoul Glaber qui évoquent l'hérésie, deux récits sur quatre l'associent à un venin et, dans tous les cas, ses acteurs sont présentés comme diaboliques. La propagation de l'hérésie dans la chrétienté correspond alors à la diffusion d'un venin, l'image de la semence achève d'en faire une évocation biologique d'autant plus effrayante que ce germe est mortifère.

Dans un autre registre, Pierre Damien (c. 1007-1072) fait de sa plume l'un des fleurons de la littérature polémique non seulement contre les hérétiques mais contre le clergé déviant que la réforme grégorienne tentait de ramener dans le giron de l'Église. Sa lettre 31 de l'édition Reindel, brocarde abondamment le clergé sodomite à travers les métaphores toxiques : ils sont les auteurs de crimes vénéneux ${ }^{9}$. Il use aussi d'une autre image pour souligner que le venin de la fausse piété se dissimule sous le miel ${ }^{10}$. L'hérésie est comme la morsure du serpent qui ôte les sens, enlève la mémoire, obscurcit l'esprit et provoque l'oubli de Dieu ${ }^{11}$. Au sein de l'Église se trouve instillé le venin de pratiques libidineuses et pestilentielles qui rappellent l'image sulfureuse de Gomorrhe ${ }^{12}$. Dans cette correspondance, l'emploi

\footnotetext{
${ }^{7}$...veneno sue nequitie plures infecit. Quod etiam seminarium nequam in pluriores hi qui susceperant toto conamine spargere nitebantur (ibid., p. 188-189).

${ }^{8}$ Sur l'ambiguïté de la génération démoniaque, voir M. van der Lugt, Le ver, le démon et la vierge. Les théories médiévales de la génération extraordinaire, Paris, Les Belles Lettres, 2004.

${ }^{9}$ Ut autem diabolicae machinationis argumenta non lateant, sed quae in officina veteris malitiae inter suos secretarios fabricat, in lucem me pallificante procedant, illud absconsum iri non patior, quod quidam huius veneno criminis satiati, dum quasi ad cor redeunt, ne reatus ad aliorum notitiam prodeat, inter se invicem confitentur et, dum hominum faciem erubescunt, qui reatus auctores existunt, ipsi iudices fiunt et indiscretam indulgentiam, quam sibi quisque affectat impendi, gaudet alteri vicaria permutatione largiri (Pierre Damien, Epistulae, CLXXX, MGH Briefe IV, Teil 1-4, K. Reindel 1983-1993, lettre 31, vol 1, p. 297).

${ }^{10}$ Sicut enim melli vel quibuslibet esculentioribus cibis venenum fraudulenter inmittitur, ut dum ad comedendum alimenti suavitas provocat, virus, quod latet, facilius se in hominis interiora transfundat, ita haec subdola et mendosa commenta sacris inseruntur eloquiis, ut suspicionem effugiant falsitatis et quodam quasi melle sunt oblita, dum falsae pietatis videntur dulcedine saporata (ibid., p. 303).

${ }^{11}$ Infelici quippe animae postquam hic venenatissimus coluber dentes semel infixerit, illico sensus adimitur, memoria tollitur, mentis acies obscuratur, inmemor Dei obliviscitur etiam sui (ibid., p. 310).

${ }^{12}$ Filia quippe populi mei pessima plaga contrita est, quia anima, quae sanctae ecclesiae fuerat filia, ab hoste humani generis telo inmunditiae est crudeliter sauciata et quae in aula regis aeterni lacte sacri eloquiii tenere ac molliter educabatur, nunc veneno libidinis
} 
de la métaphore toxique est surtout là pour souligner la duplicité des coupables qui prétendent se conformer au discours de l'Église - image de la douceur et du miel pour en fait dissimuler la perversité extrême du venin. Comme le venin dont on masque le goût avec du sucre, l'hérésie et toutes les autres déviances condamnables se dissimulent derrière une belle et fausse apparence. Pierre Damien n'est pas à l'origine de cette image qui fait florès et qui réapparait dans le Livre des sentences de Pierre Lombard achevé en 1155-1157, soit près d'un siècle plus tard. Le Lombard, pour expliquer l'expression venenum sub melle latet employée à propos de l'hérésie, reprend l'épître aux Corinthiens où il est dit que Satan s'est transformé en ange de lumière. Il cite la lettre de saint Jérôme au pape Damase dans laquelle celui-ci évoquait les Ariens et des trois hypostases qu'ils mettent en concurrence avec la Trinité, montrant ainsi les liens étroits qui unissent les discours antihérétiques de la fin de l'Antiquité à ceux du Moyen Âge. Il achève en indiquant que le venin se cache dans le miel comme Satan se transfigure en ange de lumière ${ }^{13}$. La duplicité de l'hérésie, sa capacité à cacher son venin sous le miel sont, là encore, des topoi de la littérature polémique de ce temps.

Enfin, en 1139-1140, dans son livre destiné à lutter contre le mouvement de Pierre de Bruis et adressé aux archevêques d'Arles, d'Embrun, et aux évêques de Die et de Gap, l'abbé de Cluny Pierre le Vénérable utilise l'image du venin à plusieurs reprises. Dans sa lettre-dédicace, sorte d'avant-programme, l'hérésie est présentée comme un poison mortel ${ }^{14}$. Il insiste à plusieurs reprises pour souligner que Pierre de Bruis diffuse du venin ${ }^{15}$. Par ailleurs, dans sa correspondance, il fait des hérétiques des serpents venimeux ${ }^{16}$. L'importance de la métaphore du venin dans l'ouvrage ne doit pas faire oublier qu'elle s'insère dans des réservoirs d'images variés. Dans la seconde moitié du XII ${ }^{\mathrm{e}}$ siècle, les cisterciens utilisent la métaphore de la vigne du Seigneur dont il faut faire fuir les renards. Bernard de Clairvaux dénonce l'hérésie d'Arnaud de Brescia : elle n'a pas sa place dans ce bas-monde, elle est à la

pestilenter infecta in sulphureis Gomorrae cineribus tumefacta ac rigida iacere conspicitur (ibid., p. 312).

${ }^{13}$ Sed mihi credite, venenum sub melle latet: transfigurat enim se angelus Satanae in Angelum lucis (II Cor 11, 14) (Pierre Lombard, Sententiae in IV libris distinctae, livre I, dist. XXVI, c. 1, Collegii S. Bonaventurae ad Claras Aquas, Grottaferrata, 1971, t. I, p. 197, qui cite Saint Jérôme, Epist. De fide catholica ad Damasum Papam, PL 22, c. 356, CSEL 54, p. 64).

${ }^{14}$ In quibus nunc se timore occultans, nunc de ipsis audacia assumpta prodiens, quo potest decipit, quos potest corrumpit, et nunc istis nunc illis letalia venena propinat (Pierre le Vénérable, Contra Petrobrusianos hereticos, éd. J. Fearns, Turnhout, Brepols, 1968, CCCM 10, p. 3)

${ }^{15}$ veneno infecta (ibid., p. 10); sed, ne moram faciam, spiret iam serpens sibila sua, et quibus diu tumet, venena profundat (ibid., p. 68) ; veneniferum dogma (ibid., p. 87) ; sic etiam nostri temporis hereticorum pectora venenata transfodiat (ibid., p. 154).

${ }^{16}$ Mirum sane unde surda heresis aliquando emergere potuit, cum sicut carnem sic et animam salvatoris, prophetica, evangelica, apostolica que vox ita pari et pene continuo clamore loquatur, ut vel aures non habere, vel eas more venenatae aspidis opturasse eam non exaudiens videatur (Pierre le Vénérable, Epistulae, G. Constable, Harvard Historical Studies, LXXVIII, 1967, p. 123). 
fois miel et doctrine vénéneuse, elle prend l'apparence d'une colombe à la queue de serpent $t^{17}$. Pour Berverley Maine Kienzle, à la métaphore animale s'ajoutent la démonisation, la pollution, le renversement de l'ordre social et l'Apocalypse, autant d'images destinées à éclairer de leur sombre lumière le danger hérétique ${ }^{18}$.

Ainsi, l'image du poison a plusieurs usages dans ces quelques sources des $\mathrm{XI}^{\mathrm{e}}$ et $\mathrm{XII}^{\mathrm{e}}$ siècles. Elle est très souvent associée au mal et au diable, celui qui désunit, se met en travers - dia-bolos - et constitue un danger ${ }^{19}$. L'hérésie venimeuse est destinée à se répandre dans l'ensemble de la communauté des chrétiens pour la détruire. Les hérétiques, sodomites et autres déviants se dissimulent: comme le poison, ils ne sont pas visibles mais sont destinés à se répandre dans la communauté des fidèles - une sorte de corps ou de macrocosme pour compromettre son équilibre et sa santé. L'organisme est en effet menacé par le poison qu'il a contracté, trompé par son apparente douceur mielleuse et menacé par ce germe qui, comme un virus, peut semer la mort. La contamination par une maladie contagieuse est assimilée à un empoisonnement. Selon Robert I. Moore, c'est précisément dans la seconde moitié du XII ${ }^{\mathrm{e}}$ siècle qu'a lieu cette transformation dans l'évocation de l'hérésie. Jadis assimilée à la folie elle est désormais évoquée comme une maladie : lèpre, cancer ou peste ${ }^{20}$.

Comment comprendre le développement de cette image et pourquoi a-t-elle été si étroitement associée à l'hérésie?

\section{Le serpent vénéneux dans l'hérésiologie de la fin de l'Antiquité}

Comme le Livre des sentences de Pierre Lombard a permis de le souligner, les discours anti-hérétiques plongent leurs racines dans la littérature polémique de la fin de l'Antiquitée ${ }^{21}$. Pour comprendre les liens qui existent entre le poison et l'hérésie, il convient de remonter au livre de la Genèse et aux Évangiles. Le serpent qui tenta Adam et Ève ${ }^{22}$ a très vite été assimilé au diable et à Satan ${ }^{23}$. Martine Dulaey

${ }^{17}$ Arnaldus de Brixia, cuius conversasio mel et doctrina venenum, cui caput columbae, cauda scorpionis est, quem Brixia evomuit, Roma exhorruit, Francia repullit, Germania abominatur, Italia non vult recipere, fertur esse vobis cum videte, quaseo ne vestra auctoritate plus noceat (Bernard de Clairvaux, Lettres, Epist. 196, pars 1, dans Bernardi Opera, éd. J. Leclercq et H. M. Rochais, 1974, vol. 7, p. 51).

${ }^{18}$ B. M. Kienzle, Cistercian heresy and Crusade in Occitania (1145-1229). Preaching in the Lord's Vineyard, Woodbridge, Suffolk York Medieval Press, Boydell and Brewer, 2001.

${ }^{19}$ Sur la diabolisation des hérétiques, voir: N. Cohn, Europe's Inner Demons, an Inquiry Inspired by the Great Witch-Hunt, Sussex University Press, 1975, chapitres 2 et 3; A. Vauchez, «Diables et hérétiques : les réactions de l'Église et de la société en Occident face aux mouvements religieux dissidents des la fin du $\mathrm{X}^{\mathrm{e}}$ au début du XII $\mathrm{X}^{\mathrm{e}}$ siècle » dans Santi $e$ demoni nell' alto Medioevo Occidentale (secoli V-XI), Spoleto, 1989 (Settimane di Studio del Centro Italiano di Studi sull' alto Medioevo XXXVI), p. 573-601.

${ }^{20}$ R. I. Moore, «Heresy as desease », Medievalia Lovaniensia, 1976, p. 1-11.

${ }^{21}$ Sur les racines antiques de l'hérésiologie médiévale, voir: G. Lobrichon, «Arras 1025, ou le vrai procès d'une fausse accusation» dans Inventer l'hérésie, op. cit., p. 67-86 et l'ensemble du recueil ; J.-L. Biget, «Réflexions sur 'l'hérésie' dans le midi de la France au Moyen Âge », Heresis 36-37 (2002), p. 29-74.

${ }^{22}$ Genèse (3). 
souligne cependant les subtilités qui ont entouré la première exégèse de la figure du serpent. Celui-ci a en effet souvent été rapproché du serpent de bronze que, sur l'ordre de Dieu, Moïse attacha à un bois dressé pour guérir les fils d'Israël des morsures des serpents du désert et cette figure est associée à la crucifixion ${ }^{24}$. Malgré ces nuances qui témoignent de la charge symbolique de la figure biblique, le serpent de la Genèse reste majoritairement cantonné à la figure du diable.

L'expression «engeance de vipères », employée à plusieurs reprises dans les Évangiles pour désigner les peuples sur lesquels il s'agissait de jeter l'opprobre ${ }^{25}$, a ensuite été commentée par les Pères de l'Église. Pour Irénée, évêque de Vienne et de Lyon, dans son ouvrage intitulé Adversus haereses (vers 180), l'expression désigne ceux qui n'obéissent pas à l'Évangile ${ }^{26}$. L'identification du serpent des origines et du diable peut favoriser une application plus stricte de l'image du serpent à l'hérésie. C'est en effet un thème constant de l'hérésiologie depuis Justin (†165) que d'attribuer aux hérésies une origine démoniaque. Les gnostiques, en rendant apostats leurs auditeurs, se comportent comme le serpent qui trompa $\mathrm{Eve}^{27}$. Il y a une analogie entre le serpent du Paradis et les hérétiques. Eusèbe de Césarée (v. 265340), dans l'Histoire ecclésiastique, indique que, « parmi les hérétiques, les uns à la manière des serpents venimeux, se glissaient en Asie et en Phrygie en se glorifiant de Montan comme du Paraclet $»^{28}$.

L'extension de l'image du serpent à celle du venin de l'hérésie a été réalisée de manière systématique par Épiphane de Salamine dans son Panarion, ou «Boîte à remèdes », écrit entre 374 et $377^{29}$. Dans sa préface, il indique :

Comme nous devons vous indiquer le nom des hérésies, vous révéler leurs pratiques criminelles, semblables à des venins et à des poisons et y adapter en même temps des antidotes qui serviront de remèdes pour ceux qui ont été mordus et de préventif pour ceux qui sont sur le point de tomber dans ce malheur, nous rédigeons la préface que voici pour les gens qui désirent s'instruire en l'interprétant comme un Panarion ou boîte à remèdes pour gens mordus par les bêtes venimeuses ${ }^{30}$.

Dans les trois livres de son Panarion, plus de quatre-vingt hérésies sont décrites à travers l'évocation de bêtes malfaisantes ou de serpents. Épiphane imite

\footnotetext{
${ }^{23}$ Livre de la Sagesse (2, 24), Apocalypse (12,9) et $(20,2)$.

${ }^{24} \mathrm{M}$. Dulaey, "Des forêts de symboles ». L'initiation chrétienne et la Bible (I ${ }^{e r}-V I^{e}$ siècles), Paris, le Livre de Poche, 2001, p. 225, qui cite «Dans le serpent de bronze a été figuré mon serpent, le bon serpent; un bon serpent qui, de sa bouche, répandait les remèdes au lieu du venin » (Ambroise, Sur le psaume 118, 6, 15).

${ }^{25}$ Jean-Baptiste s'adresse aux Sadducéens et aux Pharisiens (Matth. 3, 7) ; Jean-Baptiste s'adresse au peuple (Luc 3, 7); Jésus s'adresse aux Pharisiens (Matth. 12, 34); Jésus s'adresse aux Scribes et aux Pharisiens (Matth. 23, 33).

${ }^{26}$ progeniem viperarum (Irénée de Lyon, Adversus haereses IV, 41, éd. A. Rousseau (SC 100), 1965, p. 989).

${ }^{27}$ Ibidem, IV, 4, p. 386- 388.

${ }^{28}$ Eusèbe, Histoire ecclésiastique II, 1. V, 14, éd. G. Bardy (SC 41), 1983, p. 45.

${ }^{29}$ A. Pourkier, L'hérésiologie chez Épiphane de Salamine, Paris, Beauchesne, 1992.

${ }^{30}$ Épiphane de Salamine, Panarion, Préface citée dans ibid., p. 77.
} 
en cela les zoologues et les botanistes comme Nicandre qui a écrit des vers sur les bêtes sauvages ou les serpents, ou Dioscoride qui a traité de la matière des racines et des plantes ${ }^{31}$. Chaque hérésie est ainsi comparée à un serpent très précis, une taupe ou une musaraigne. Ainsi, Épiphane se fait l'héritier d'une tradition qui a associé le serpent de la Genèse au diable et aux hérésies mais il développe de manière approfondie la métaphore du venin de l'hérésie dans un ouvrage destiné à en définir les remèdes, c'est en tous cas le sens du terme Panarion. Cette association du mal et de sa guérison rappelle la tradition grecque du pharmakon qui signifie à la fois poison et remède ${ }^{32}$. Cette double acception s'applique d'ailleurs particulièrement bien aux hérétiques qui paraissent ressembler à ces victimes expiatoires mises à mort dans la cité grecque afin d'en restaurer l'unité menacée par une crise momentanée ${ }^{33}$. Dans le cas des hérétiques, il n'est pas question de les mettre à mort à la fin de l'Antiquité, même si la question se pose au XIII ${ }^{\mathrm{e}}$ siècle avec la naissance de l'Inquisition.

Le creuset idéologique des premiers siècles a donc été favorable à la diabolisation des hérétiques ${ }^{34}$, le développement de l'image du venin de l'hérésie date de cette époque et elle est reprise de manière systématique aux $\mathrm{XI}^{\mathrm{e}}$ et $\mathrm{XII}^{\mathrm{e}}$ siècles. Mais ne peut-on pas aller plus loin et voir dans la diabolisation des déviants au sens large, allant même jusqu'aux pécheurs, une sorte de prise de possession venimeuse?

\section{Le pécheur, le possédé et le poison}

Comme les exemples extraits des Histoires de Raoul Glaber le suggèrent, le venin est aussi associé à la possession diabolique ${ }^{35}$. L'analogie entre le possédé et la victime du poison transparaît à travers certaines descriptions; les effets du poison étant parfois même assimilés à la possession par le démon ${ }^{36}$. Le démoniaque peut avoir ingéré le diable comme une substance nocive. Ainsi, dans les Dialogues de Grégoire le Grand (v. 540-604), une moniale ingère le diable avec une laitue qu'elle avait oublié de bénir ${ }^{37}$. Le corps de la victime est immédiatement torturé de douleur

\footnotetext{
${ }^{31}$ Ibidem, p. 77.

${ }^{32}$ F. Collard, «Pharmaca aut venena, pharmaca sive venena. Réflexions sur la perception d'une contiguïté durant le Moyen Âge latin», Le corps à l'épreuve. Poisons, remèdes et chirurgie : aspects des pratiques médicales dans l'Antiquité et au Moyen Âge, dir. F. Collard, E. Samama, Langres, D. Guéniot, 2002, p. 105-122. Dans le mythe de Dionysos, l'ambiguïté du mot apparaît bien puisqu'il est appliqué au vin qui provoque la mania, celui qui l'absorbe devient mainomenos, c'est-à-dire possédé (J.-P. Vernant, «Le Dionysos masqué des Bacchantes d'Euripide », La Grèce ancienne. 3, Rites de passage et transgression, J.P. Vernant, P. Vidal-Naquet, Paris, Seuil, 1992, p. 253-287).

${ }^{33}$ R. Girard, La violence et le sacré, Paris, Grasset, 1972, p. 144.

${ }^{34}$ E. Pagels, L'origine de Satan, éd. fr. Paris, Bayard, 1997.

${ }^{35}$ Sur le sujet, voir: F. Chave-Mahir, Une parole au service de l'unité. L'exorcisme des possédés dans l'Église d'Occident $X^{e}-X I V^{e}$ siècles, Thèse dactylographiée, dir. N. Bériou, Université Lyon II, 2004, à paraître aux éditions Brepols.

${ }^{36}$ F. Collard, Le crime de poison, op. cit., p. 73.

${ }^{37}$ Grégoire le Grand, Dialogues I, 4, 7, éd A. de Vogüé et P. Antin (SC 251), Paris, Cerf, 1979, p. 43-45.
} 
et son malaise est bien physique. Des récits, présents dans les recueils d'exempla du $\mathrm{XIII}$ siècle, le font apparaître. Une femme possédée pâlit et les veines de son cou gonflent à l'écoute de versets du Deutéronome ${ }^{38}$. Le diable est visible sous la peau d'une autre possédée à l'approche du livre des Évangiles ${ }^{39}$. La totalité du corps est atteinte lors de cette affection, comme le laissent supposer plusieurs formules liturgiques de l'exorcisme, en particulier dans le pontifical romano-germanique du $\mathrm{X}^{\mathrm{e}}$ siècle. Toutes les parties du corps y sont successivement nommées comme autant de lieux dont il faut faire partir le diable ${ }^{40}$. Certains formulaires mentionnent explicitement que le diable qui envahit le possédé est un venin ${ }^{41}$. Enfin, le diable est immanquablement expulsé dans un violent vomi ou un flux de ventre que provoque, aussi, l'infection venimeuse ${ }^{42}$. La presque totalité des récits d'exorcisme mentionnent cette expulsion du diable. Plusieurs miracles racontent l'émission de $\operatorname{sang}^{43}$, d'animaux ou d'insectes ${ }^{44}$. La sortie du diable par la bouche est, d'ailleurs, le code iconographique qui permet, dans tous les cas, de reconnaitre la possession dans les représentations figurées médiévales. Contrairement à l'atteinte toxique cependant, rarissimes sont les crises de possession qui provoquent la mort, c'est un état très souvent transitoire et la guérison miraculeuse du sujet permet de valoriser l'action liturgique mise en œuvre contre lui. Cette action a donc quelque parenté avec la pratique médicale puisque l'exorcisme peut être vu comme une sorte d'émétique, un vomitif utilisé dans les cas d'intoxication. Mais l'exorcisme ne

\footnotetext{
${ }^{38}$ Thomas de Cantimpré, Bonum universale de apibus, éd. Colvenere, Douai, 1627, II, 57, 67.

${ }^{39}$ Giraud de Cambrai, Gemma Ecclesiastica Opera II, éd. J. S. Brewer, Londres, Longman, 1868, c. 18, p. 54, voir aussi, Thomas de Cantimpré, Bonum universale, op. cit., II, 36, 4.

${ }^{40}$ Domine, sancte pater, omnipotens eterne Deus, expelle diabolum ab homine isto $N$., de capite, de capillis, de vertice, de cerebro, de fronte, de oculis, de auribus, de naribus, de ore, de lingua, de sublingua, de gutture, de collo, de pectore toto, de corde, de corpore toto, de omnibus membris, de compagninibus suorum membrorum intus et foris, de ossibus, de venis, de nervis, de sanguine, de sensu, de cogitationibus, de verbis, de omnibus operibus suis, de inventute, de omni conversatione eius hic et in futuro, ut operetur in eo virtus Christi Iesu filii Dei vivi et altissimi, qui regnat in saecula seculorum. Amen (C. Vogel et R. Elze, Le pontifical romano-germanique du $X^{e}$ siècle, tome II (ST 227), p. 192).

${ }^{41}$ Omnia nequissimi spiritus ab eo venena depelle et salutari baptismi tui gratia adimple, ut tui muneris perceptione in aeterna vita valeat exultare. Per., Impositio manuum super energuminum catezizatum (ibid., p. 192); Domine Iesu Christe, cui omnia subiecta sunt, quem omnis natura et omnis creatura pavescit recedens in semetipsam, cum te ad auxilium invocemus, cuius audito nomine serpens mansuescit et draco fugit et silet vipera et rubeta statim torpescit, scorpio extinguitur, regulus vincitur et spalangius nichil noxium operatur et omnia venenata et adhuc ferotiora repentia et animalia noxia tenebrantur et omnes adversae naturae radices inanescunt. Tu extingue hunc venenosum serpentem, virus eius extingue, operationes eius mortifica, quas in se habet <vires> evacua et da in conspectu tuo omnibus his, quos tu creasti, oculos ut videant, aures ut audiant gratiam tuam, cor ut magnitudinem tuam intellegant, salvator mundi, qui cum patre (ibid., p. 198-199).

${ }^{42}$ F. Collard, Le crime de poison, op. cit., p. 80 et suiv.

${ }^{43}$ Miraculorum S. Eutropio, AASS avril III, n. 22, p. 749 ; Bouillet, Miraculorum Sancte Fidis, Paris, 1897, p. 592, 295.

${ }^{44}$ Les miracles de saint Benoît, éd. de Certain, Paris, 1858, I, p. 69 ; et ibid., II, p. 114.
} 
prévoit pas vraiment l'administration d'une substance matérielle au possédé, si ce n'est parfois de l'eau bénite, du sel ou un jeûne prolongé ${ }^{45}$. Ce sont surtout l'action des signes de croix, des bénédictions, des prières efficaces qui viennent à bout de la présence du démon. Tels sont les vrais remèdes, mais nous y reviendrons.

Les liens entre la possession et les métaphores venimeuses sont aussi visibles dans une source historique bien différente des formulaires d'exorcisme, et plus tardive. En effet, à l'occasion de certains sermons du XIII ${ }^{\mathrm{e}}$ siècle des prédicateurs, s'adressant à leur public de pécheurs en puissance que sont tous les fidèles, emploient les images de la possession et du venin pour leur faire prendre conscience de la gravité des forfaits commis ou à venir. Lors du second dimanche de l'Avent, Jean d'Abbeville, archevêque de Besançon et cardinal $(\dagger 1237)$, explique que le pécheur est aveugle et muet. Il le compare à un démoniaque qui n'a pas toute sa raison, qui est comme la monture du diable et qui, surtout, ne décèle pas l'amertume venimeuse du péché :

En outre, le démoniaque croit et se réjouit tant qu'il ne sent pas l'affliction qu'il porte. Il est comme ceux qui se réjouissent des choses les pires et qui croient faire le mal. Ils se plaignent du miel dans le fiel, de la douceur dans le péché, comme le dit Job : 'Des buissons on les entendait braire et ils s'entassaient sous les chardons' (Job 30, 7). Le péché ne leur est pas amer. Amère est la nourriture que l'âme ne peut avaler sans mourir, comme le dit Job : 'Peut-on goûter à ce dont le goût apporte la mort ?' (Job, 6, 6). Mais nombreux sont comme le porc, c'est-à-dire un animal impur selon la Loi, car il ne rumine pas : ils avalent le péché sans le mâcher et comme le dit Job: 'Ils boivent l'iniquité comme l'eau' (Job 15, 16). Le péché est avalé mais non poli par les dents. Ainsi, comme le médecin prend des pilules amères dans le brouillard pour vite qu'on les avale sans les mâcher afin de ne pas sentir l'amertume, ainsi le diable s'empare du venin du péché dans le brouillard et non dans la douceur de la vérité. Mais si l'homme avait mâché ce voile, il aurait senti l'amertume et aurait mis en fuite le péché ${ }^{46}$.

45 Ainsi, le pontifical romano-germanique comporte parmi de nombreuses formules liturgiques une sorte de recette médicale contre la possession. Ce «jeûne à imposer au démoniaque » consiste à manger du pain non levé avec de l'eau bénite et du sel, pendant les cinq premières semaines, ensuite le possédé aura droit à un peu de lard et de la cervoise. C'est alors que le prêtre lui donne un vomitif composé d'eau, de sel bénits et d'absinthe (det illi presbiter aquam benedictam cum sale sanctifucato bibere et cum absinthio mixta donec evomat) (C. Vogel, R. Elze, Le pontifical romano-germanique, op. cit., p. 220-221).

${ }^{46}$ Preterea demoniacus gaudet et exultat tanquam non sentiens afflictionem quam portat et tales sunt qui exultant in rebus pessimis et gaudent cum male fecerunt, qui querunt mel in felle, dulcedinem in peccato, de quibus Iob XXX: Qui letantur in huiuscemodi et esse sub sentibus delicias reputabant. Peccato quidem nichil amarius. Amarus est cibus quem anima sine morte glutire non potest sicut dicit Iob VI : Quis gustavit quod gustatum affert mortem? Sed multi sunt velud sus, ideo immundum animal secundum Legem quia non ruminat: glutiunt enim peccatum et non masticant, et sicut dicit Iob : Bibent iniquitatem sicut acquam, glutientes non dente terentes, quia sicut medicus pillulas amaras involuit nebula ut cito gluciantur nec emasticentur, quia sapor amaritudinis ferri non posset, sic dabolus venenum peccati involuit quadam nebula et non veritate dulcedinis, sed si illud involucrum homo 
Jean d'Abbeville n'est pas le seul à croiser l'image du poison avec celle du pécheur devenu démoniaque. Dans nombre de sermons du XIII ${ }^{\mathrm{e}}$ siècle en effet, en particulier durant le temps de Carême, les prédicateurs développent ainsi l'image de la possession pour évoquer le pécheur auquel ils s'adressent. Ces métaphores sont particulièrement développées à l'occasion du troisième dimanche de Carême qui commente l'Évangile de Luc : Erat Iesus eiciens demonium ${ }^{47}$. Le pécheur est comme un possédé qui ne contrôle plus ni son corps ni son esprit. Il est environné de serpents et de bêtes venimeuses qui figurent, toujours, le démon menaçant ${ }^{48}$.

Ainsi, l'évocation de la possession, dans les récits narratifs comme dans certains sermons est liée à celle du poison. À tel point que, pour les prédicateurs du $\mathrm{XIII}^{\mathrm{e}}$ siècle, la trilogie pécheur-possession-diable (empoisonné) ne fait plus qu'un. Il est naturellement possédé ce pécheur qui ne reconnaît pas l'amertume du venin car il ne sait méditer, savourer et mâcher les paroles de l'Évangile. L'hérétique, comme possédé, diffuse son venin dans l'Occident, et il est directement associé à lui.

\section{Quel antidote au venin de l'hérésie et du péché?}

Filant la métaphore jusqu'au bout, les auteurs du Moyen Âge n'ont pas hésité à présenter leur action pastorale comme un contre-poison, le Mal dans son ensemble devenant une sorte de pharmakon ${ }^{49}$. Comme dans le Panarion d'Épiphane de Salamine, Pierre le Vénérable présente l'œuvre apologétique dans laquelle il s'est engagé, comme un antidote contre le poison de l'hérésie ${ }^{50}$.

De manière générale, l'écoute du sermon et le strict respect des principes de l'Église sont des remèdes efficaces. C'est ainsi l'argument du sermon de Jean d'Abbeville déjà cité. Il indique que la grâce de l'Esprit Saint est comme une thériaque contre le venin du péché ${ }^{51}$. La thériaque est un électuaire employé contre la

masticaret sentiret amaritudinem et refugeret a peccato (Jean d'Abbeville, Sermo in secunda dominica adventus, dans L. J. Bataillon, «Early Scholastic and Mendicant Preaching as Exegesis of Scripture», dir. M. D. Jordan, K. Emery, Ad litteram. Authoritative Texts and Their Medieval Readers, University of Notre Dame, London, 1993, p. 181-182).

${ }^{47}$ F. Chave-Mahir, «Liturgie de l'exorcisme et sermons au XIII ${ }^{\mathrm{e}}$ siècle. Les images de la possession dans les sermons du troisième dimanche de Carême », dans Prédication et liturgie au Moyen Âge, dir. N. Bériou et F. Morenzoni, Turnhout, Brepols, 2008, p. 237-253.

${ }^{48}$ Lamie sunt qui habent faciem hominis et lac venenosum (Pierre de Reims, Dominica III in Quadragesima, dans L. J. Bataillon, «Early Scholastic», op. cit., p. 190).

${ }^{49}$ Même si le terme n'a aucun équivalent en latin, il y a une subtile continuité entre les civilisations grecque et latine à propos du double sens du poison, F. Collard, «Pharmaca aut venena, pharmaca sive venena », op. cit., p. 111.

${ }^{50}$ Hii plane de antidoto venenum, de salute exitium, de vita mortem stulto sibi commercio emunt et cum apostolus dicat 'expectantes beatam spem, et spe salvi facti sumus', cum spe salvari debuerint, contra spem spe pereunt, quia qualiter uti spe debeant non attendunt (Pierre le Vénérable, Contra Petrobrusianos, op. cit., p. 160).

${ }^{51}$ Siquidem toxicum magis venenosum et magis noxium probatur per virtutem tyriace, quia contra pessimum toxicum oportet haberi tyricam optimam; scitis autem quod peccatum Ade acutissimum fuit toxicum infectione duplici, scilicet miseria pene et vitio culpe; tyriaca autem est gratia Spiritus sancti, que etsi in viris sanctis habundaverit ad purgationem culpe, tamen non purgavit miseram pene; nam etsi in humana Christi natura fuit plenissima divinitas, non 
morsure du serpent, il est donc l'anti-venin par excellence. Alexandre Nequam $(\dagger 1217)$ a, lui aussi, recours à cette image dans le De naturis rerum ${ }^{52}$, peut-être Jean d'Abbeville, mort vingt ans plus tard, s'inspire-t-il de lui ? Certainement en tous cas que l'image est suffisamment suggestive pour assurer son succès. Pour Guillaume Peyraut $(\dagger 1271)$, dans un sermon pour le dixième dimanche après la Trinité, ce sont les bénédictions qui sont des remèdes contre le poison ${ }^{53}$. Selon Ranulphe de la Houblonnière, la confession agit contre les substances toxiques du péchés4. Quant à Jacques de Voragine, il renverse le sens des toxiques en présentant le jeûne comme un poison contre le diable ${ }^{55}$. Tout l'arsenal des gestes, des paroles et des pratiques destinés à mettre le diable en fuite des corps possédés se retrouve dans ces évocations métaphoriques du Mal et du péché.

Ainsi, l'Église emploie des métaphores venimeuses très suggestives de la menace qui plane sur les individus et sur l'ensemble de la communauté des fidèles, celle d'une substance toxique transmise d'un être à l'autre pour finalement compromettre l'ensemble de la chrétienté, à travers les hérétiques mais aussi les pécheurs. Le clergé, armé de gestes, de paroles et de sacrements, est en mesure de la

tamen fuit per hanc tyriacam purgata misera pene (Jean d'Abbeville, Sermo in secunda dominica adventus, dans L. J. Bataillon, «Early Scholastic and Mendicant Preaching», op. cit., p. 182).

${ }_{52}$ Alexandre Nequam, De naturis rerum, éd. T. Wright, Londres, 1863, II, 109, p. 190, cité par F. Collard, Le crime de poison, op. cit., p. 95.

${ }^{53}$ Tertio ex hoc quod mala ei sunt bona, ipse enim nihil habet malum juxta illud Ecclesiasta 8. Qui custodit praceptum non experietur quicquam mali Ad Rom. 8. Scimus quoniam diligentibus Deum omnia cooperantur in bonum. Quod aliis esset venenum, est ei remedium. Talis etiam benedictus est quasi infinitis benedictionibus. Unde Deut. 28. Venient super te omnes benedictiones ista et apprehendent te, si tamen pracepta ejus audieris. Benedictus tu in civitate, etc. et paulo post: Emittet Dominus benedictionem super cellaria tua, et super omnia opera manuum tuarum (Guillaume Peyraut, sermon pour le dixième dimanche après la Trinité, dans Guillaume d'Auvergne, Opera omnia, tome II, 1, Orléans et Paris, 1674, p. 106, col. B).

${ }^{54}$ Secundo, fit vera sanctificatio Deo per veram confessionem. Videtis quod, quando unus homo, vel mulier comedit aliquod invenenatum, non potest habere bonum nec pacem donec evomerit. Sed non est ordura tanta, neque venenum ita mortiferum sicut peccatum. Sed istud evomitur in sancta confessione (Ranulphe de la Houblonnière, sermon pour le second dimanche de Carême, dans N. Bériou, La prédication de Ranulphe de la Houblonnière. Sermons au clercs et aux simples gens à Paris au XIII ${ }^{e}$ siècle, Paris, Études Augustiniennes, 1987, tome II, p. 82).

${ }_{55}^{5}$ Diabolus autem prohibet ieiunium, quia est sibi venenum. Dicit enim Ambrosius quod sputum hominis ieiuni est serpenti venenum, per quod datur intelligi quod venenum serpenti infernali est ieiunium (Jacques de Voragine, troisième dimanche de Carême, sermon 2, R. Clutium, 1759, p. 278). Le crachat d'un homme à jeun est comparable au venin du serpent, (voir aussi le sermon de Ranulphe de la Houblonnière dans N. Bériou, La prédication de Ranulphe de la Houblonnière, op. cit., p. 201). Cette image est un emprunt à saint Ambroise : Ieuni hominis sputum, si serpens gustaverit, moritur. Vides quanta vis ieiunii sit ut et sputo suo homo terrenum serpentem interficiat et merito spiritualem (Ambroise, Hexameron VI, 4, éd. Schenkl, CSEL 32/1, p. 223). 
purger de ce mal comme de bons médecins. Les exorcistes, grade ecclésiastique par lequel tous les clercs sont passés, ne sont-ils pas qualifiés, au cours de leur ordination, de medici ecclesie ${ }^{56}$ ?

La métaphore du poison est bien une image «intentionnelle» dans les discours anti-hérétiques des $\mathrm{X}^{\mathrm{e}}-\mathrm{XII}{ }^{\mathrm{e}}$ siècles et dans la prédication aux fidèles du $\mathrm{XIII}^{\mathrm{e}}$ siècle. Dans le premier cas, il s'agit de jeter l'opprobre sur un groupe à un moment où l'Église tente de renforcer son unité face aux déviances religieuses. Dans le second cas, cette image efficace a pour but d'appuyer un discours disciplinaire directement adressé aux simples pécheurs. La métaphore venimeuse n'innove pas. Elle prend place dans un vocabulaire négatif inspiré d'une tératologie abondante, du diable, de la possession démoniaque, de la maladie et de la mort. Peut-être qu'en utilisant l'image du venin, les lettrés des $\mathrm{X}^{\mathrm{e}}-\mathrm{XIII}{ }^{\mathrm{e}}$ siècles pensent sans le savoir au pharmakon des Grecs, à la fois poison et remède. Car le venin ainsi évoqué dans le discours sous la forme de l'hérésie ou du péché n'est concevable que parce qu'il contient son remède : l'action apologétique de l'Église dans son ensemble.

Florence Chave-Mahir Université Lumière-Lyon II

CNRS, UMR 5648.

${ }^{56}[. .$.$] potestatem et imperium habeant spiritus immundos cohercendi et probabiles sint$ medici ecclesiae (C. Vogel, R. Elze, Le pontifical romano-germanique, op. cit., p. 17). 